\title{
RESTOS FOSILES DE TORTUGAS EN SAN BUENAVENTURA NEALTICAN, PUEBLA
}

\author{
Jorge Alfredo HERRERA-FLORES* \\ Escuela de Biología, Benemérita Universidad Autónoma de Puebla, Edificio 76, \\ Bulevar Valsequillo y Circuito CU Ciudad Universitaria, Puebla, MÉXICO. \\ *Dirección actual: Instituto de Ecología A. C. Km. 2.5 carretera antigua a Coatepec 351, \\ Congregación El Haya, Xalapa 91070, Veracruz, México. Apartado Postal 63 \\ E-mail: jorge.herrera@posgrado.inecol.edu.mx
}

Herrera-Flores, J. A. 2009. Restos fósiles de tortugas en San Buenaventura Nealtican, Puebla. Acta Zoológica Mexicana (n. s.), 25(3): 455-464.

RESUMEN. Se describe y comenta material fósil de tortugas, procedente de San Buenaventura Nealtican, Puebla. Según sus características morfológicas es referido al género Trachemys, lo que representa el primer registro fósil de este género para el estado de Puebla, además de dar a conocer una nueva localidad con herpetofauna para el Pleistoceno de Puebla.

Palabras clave: Tortugas, Fósiles, Emydidae, Trachemys, San Buenaventura Nealtican.

Herrera-Flores, J. A. 2009. Fossil turtles remains from San Buenaventura Nealtican, Puebla. Acta Zoológica Mexicana (n. s.), 25(3): 455-464.

ABSTRACT. Fossil material of turtles from San Buenaventura Nealtican, Puebla is described and commented. According to its morphologic characteristics this material is referred to the genus Trachemys, which represents the first fossil record of this genus for the State of Puebla and a new locality with herpetofauna for the Pleistocene of Puebla.

Key Words: Turtles, Fossils, Emydidae, Trachemys, San Buenaventura Nealtican.

\section{INTRODUCCIÓN}

El registro de vertebrados pleistocénicos en el estado de Puebla es abundante, esta entidad es la tercera con mayor número de sitios en México, con un aproximado de 57 localidades (Arroyo-Cabrales et al. 2002). Sin embargo, los restos fósiles de reptiles son escasos, debido a que la mayoría de los registros fósiles pertenecen a mamíferos. Hasta el momento sólo hay tres localidades de edad pleistocénica reportadas con herpetofauna en el estado de Puebla: el Cañón Tecorral en el Valle de Tehuacán (Flannery 1967), Puente de Atepetzingo en Valsequillo (Guenther 1968) y Santa Cruz Nuevo en el Municipio de Totoltepec de Guerrero (Guillette et al. 2000; Tovar-Liceaga \& Montellano-Ballesteros 2004, 2005; Tovar et al. 2007). No obstante, algunos de los registros de herpetofauna para el Pleistoceno del estado de Puebla, son dudosos porque

Recibido: 30/11/2007; aceptado: 04/09/2009. 
no muestran, ni hacen mención de material referido, además de no proporcionar descripciones detalladas (e. g. Flannery 1967; Guenther 1968).

Actualmente, el Centro Regional INAH-Puebla cuenta una colección paleontológica con material fósil de gran importancia que en su mayoría fue recolectado a mediados del siglo pasado por el maestro Juan Armenta, así como por investigadores alemanes quienes realizaron trabajos geológicos y paleontológicos en el área de Puebla-Tlaxcala durante la década de 1960 (Guenther \& Bunde 1973; Cruz-Muñoz 2001). No obstante, mucho de ese material fósil quedó abandonado durante varios años, por lo que gran parte aún permanece sin estudiar (Cruz-Muñoz et al. 1998). Como parte del material paleontológico del Centro Regional INAHPuebla, se encuentran resguardados fósiles de tortugas presumiblemente de edad pleistocénica, provenientes de la localidad San Buenaventura Nealtican. Este material es de gran importancia, ya que para Nealtican, sólo se conocen vestigios de asentamientos humanos prehispánicos (e. g. Seele 1973; Plunket \& Uruñuela 1999), por lo que no existe información sobre la fauna que habitó la zona en tiempos prehistóricos. Por tal motivo, el estudio y descripción de los fósiles de tortugas de San Buenaventura Nealtican son el principal objetivo de este trabajo.

\section{MATERIAL Y MÉTODOS}

Localidad y edad. San Buenaventura, Municipio de Nealtican (19 ${ }^{\circ} 03^{\prime} 6.39^{\prime}$ ' N y $98^{\circ}$ 25' 35.91" O), localizado en la parte centro oeste del estado de Puebla, a aproximadamente $23 \mathrm{~km}$ al oeste de la Ciudad de Puebla (Fig. 1). Estudios estratigráficos de la región Puebla-Tlaxcala (Erffa et al. 1976; Erffa \& Hilger 1977), señalan a la zona de San Buenaventura Nealtican como de edad cuaternaria, compuesta por depósitos piroclásticos y cenizas volcánicas. Estudios geológicos recientes (Siebe $e t$ al. 1995; Macías 2005), indican que ésta área está conformada por remanentes de erupciones plinianas del Volcán Popocatépetl ocurridas durante los últimos 20,000 años. Sin embargo, la edad de los fósiles de San Buenaventura Nealtican se asigna al Pleistoceno Tardío con base en material orgánico reportado en estudios estratigráficos del volcán Popocatépetl con una edad de 14,000 años (Macías 2005).

Material examinado. Los fósiles están resguardados dentro de la Colección Paleontológica del Centro Regional INAH-Puebla, con los números de catálogo CRINAHP-1841 y CRINAHP-1842. Ambos ejemplares se encontraban cubiertos por una capa de sedimento, por lo que se limpiaron utilizando agua y cepillo de dientes, posteriormente se consolidaron, inyectándoles pegamento adhesivo instantáneo en los agrietamientos. Terminada la preparación de los especímenes, se procedió a tomar las medidas de los diferentes elementos (plastrón y caparazón) empleando una cinta métrica. Todas las mediciones se tomaron en milímetros. La identificación taxonómica se realizo mediante comparación directa y bibliográfica. La clasificación sistemática utilizada en este trabajo se basa en la presentada por Joyce et al. (2004). 


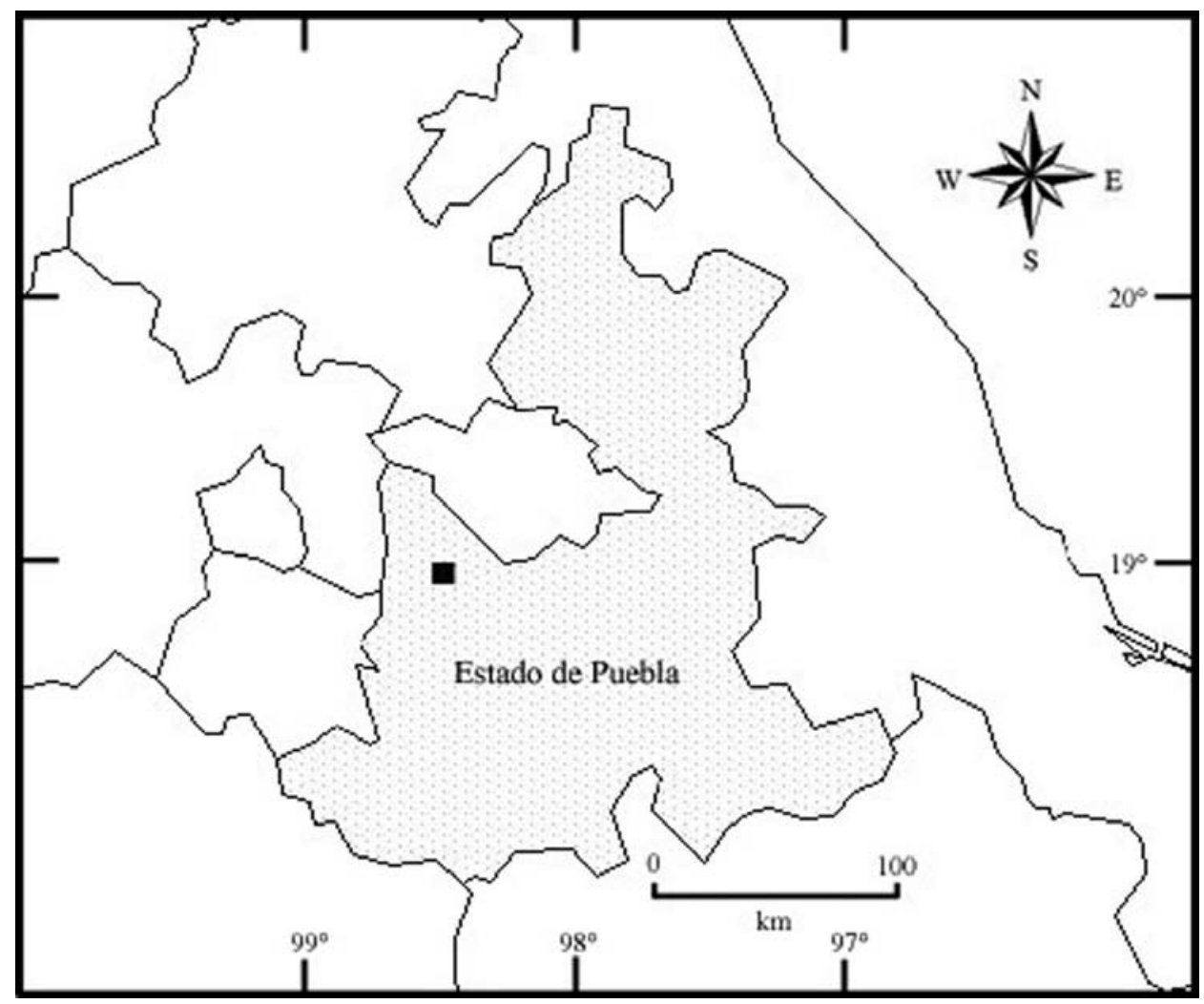

Figura 1. Ubicación geográfica de San Buenaventura Nealtican en el estado de Puebla (cuadro).

Abreviaturas. CRINAHP, Centro Regional INAH (Instituto Nacional de Antropología e Historia) Puebla.

\section{RESULTADOS}

\section{Paleontología Sistemática}

Orden Testudines Batsch 1788

Suborden Cryptodira Cope 1868

Familia Emydidae Bell 1825

Género Trachemys Agassiz 1857

Trachemys sp. 
Material. Placa izquierda del hyoplastrón (CRINAHP-1841; Fig. 2).

Descripción. Este elemento muestra una forma cuadrada, a su alrededor se logran apreciar las suturas que lo articulaban con las demás partes del plastrón y caparazón. En su costado izquierdo se observa una sutura de forma arqueada la cual contactaba con el caparazón, mientras que en el costado derecho se aprecia una sutura de forma recta, que unía a este elemento con la placa derecha del hyoplastrón. En la parte anterior derecha del hyoplastrón se observan dos suturas, la primera de forma curva, que articulaba a este elemento con el entoplastrón, la segunda es de forma recta y conectaba al hyoplastrón con el epiplastrón. En la parte anterior izquierda se observa claramente la fosa axilar del hyoplastrón, mientras que en la parte posterior se encuentra una sutura de forma recta que unía al hyoplastrón con el hypoplastrón. La longitud antero-posterior es de $142 \mathrm{~mm}$, mientras que el ancho mayor conservado es de $140 \mathrm{~mm}$.

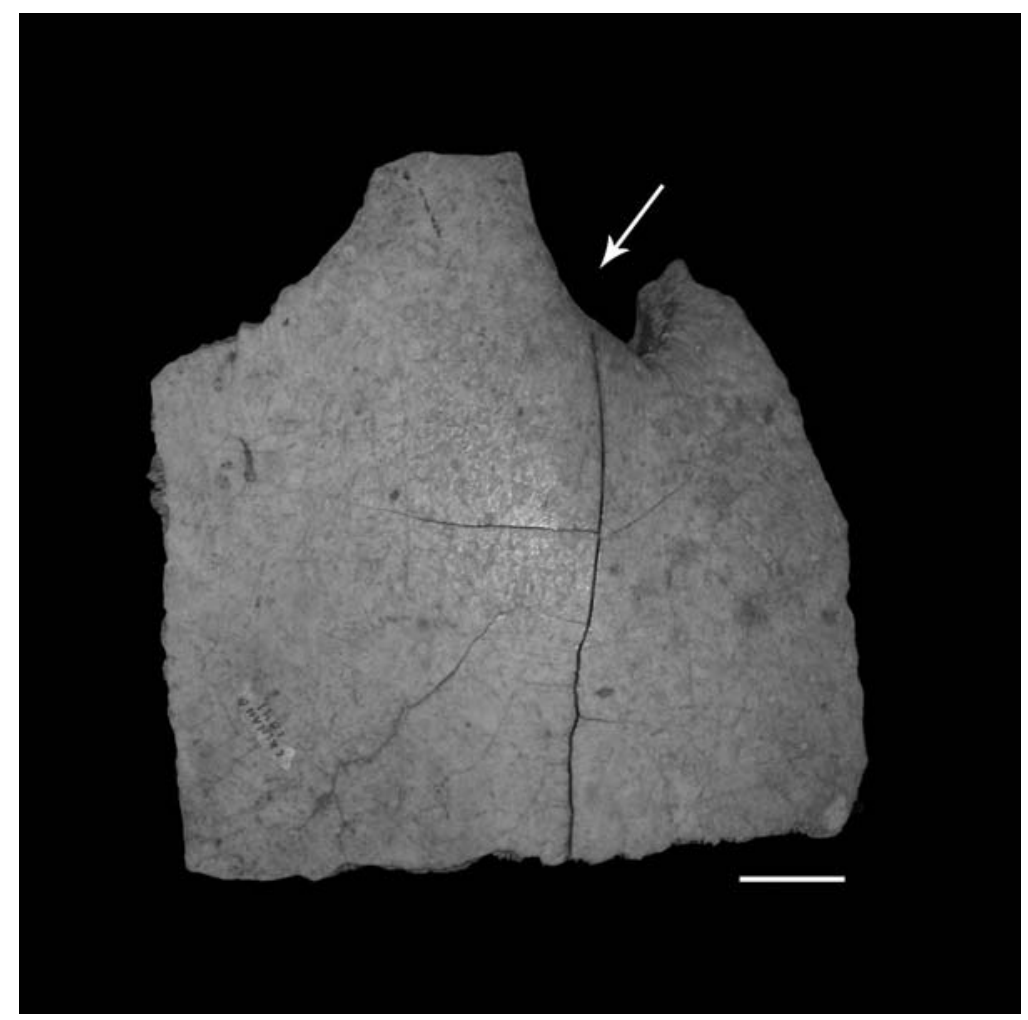

Figura 2. Ejemplar CRINAHP 1841. Hyoplastrón izquierdo. Nótese las suturas de contacto alrededor de todo el elemento, así como la fosa axilar del plastrón (flecha). Línea horizontal = $20 \mathrm{~mm}$. 
Material. Fragmento de caparazón (CRINAHP-1842; Fig. 3).

Descripción. Fragmento de caparazón que pertenece al costado derecho. Presenta una forma oval, moderadamente arqueada, dorsalmente se observa ligeramente aplanado (Fig. 3 a) y en vista ventral se aprecia la presencia de siete costillas (Fig. 3 b). Alrededor de todo el costado derecho de este fragmento se observa la sutura que lo unía con las placas periféricas, mientras que en el costado izquierdo se logra apreciar que este elemento se fracturó sagitalmente dividiéndose del resto del caparazón. Las suturas que separan a las placas óseas del caparazón no se observan de forma clara en el ejemplar, posiblemente como consecuencia del proceso de mineralización ocurrido durante la fosilización o del desgaste por intemperismo, y no por una patología presente en el ejemplar. La longitud antero posterior es de $308 \mathrm{~mm}$, y su ancho mayor conservado es de $106 \mathrm{~mm}$.

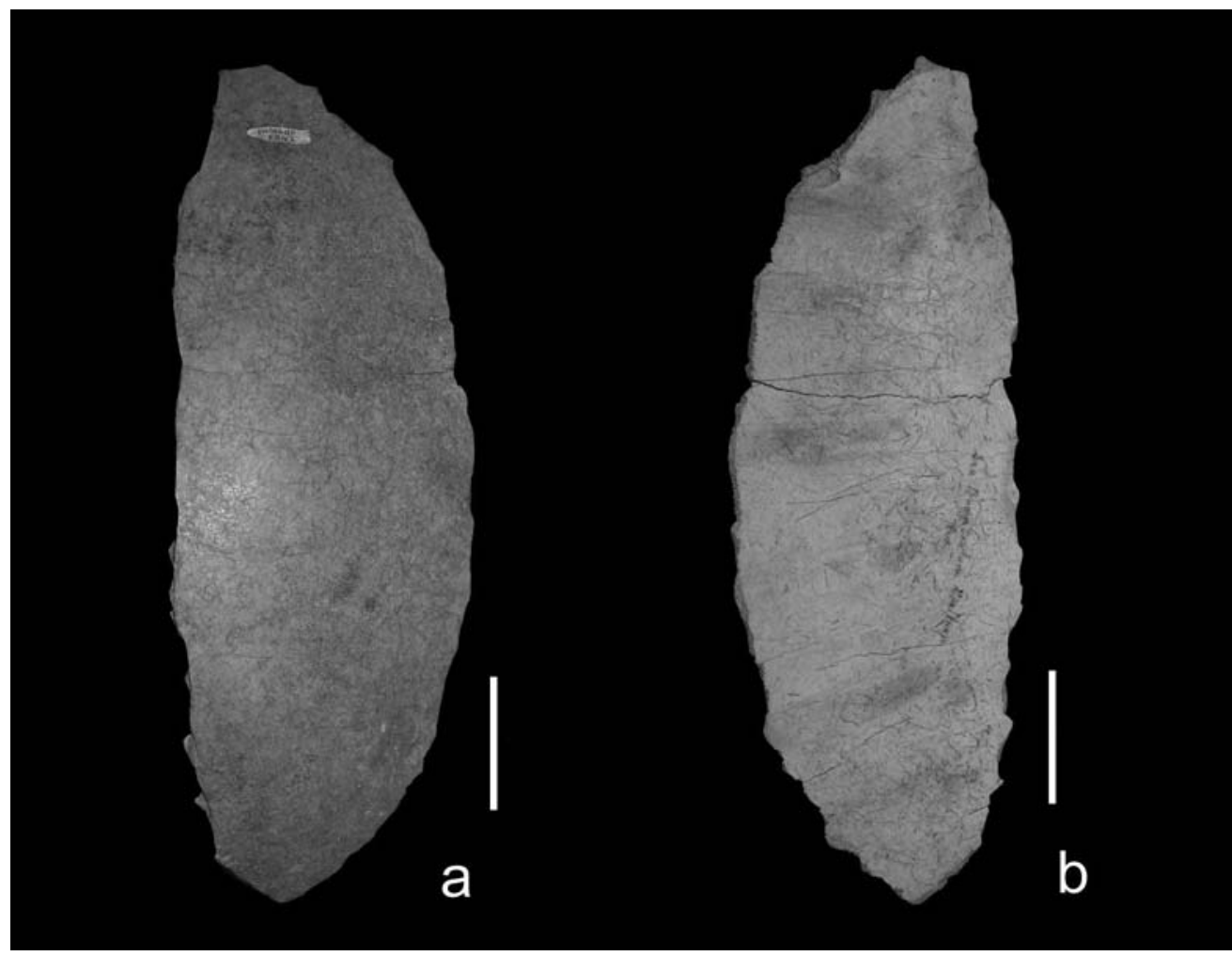

Figura 3. Ejemplar CRINAHP 1842. Fragmento de caparazón. a) Vista dorsal b) Vista ventral. Líneas verticales $=50 \mathrm{~mm}$. 


\section{DISCUSIÓN}

Determinación taxonómica. Los fósiles de Nealtican difieren morfológicamente de otras tortugas comunes en el registro fósil de México como la familia Kinosternidae, debido a que estas tortugas son de tamaño pequeño y caparazón fuertemente arqueado. En México, sólo se conocen fósiles del género Kinosternon que carece de entoplastrón (Ernst \& Barbour 1989), por lo que no es posible relacionar a los fósiles de San Buenaventura Nealtican con Kinosternidae. Por otro lado, la familia Testudinidae representada por fósiles de los géneros Geochelone, Gopherus y Testudo, difieren de los especímenes de Nealtican, debido a que la fosa axilar del plastrón en Testudinidae es corta. Además, el caparazón de los testudínidos es abombado y con disminución de tamaño en las costillas (Hay 1908).

Por su parte, al comparar a CRINAHP-1841 y CRINAHP-1842 con la familia Emydidae, se tiene que ambos elementos comparten características que los relacionan con esta familia y en especial con el género Trachemys. Las características que apoyan la asignación de los ejemplares de Nealtican con Trachemys, son la típica forma cuadrada con una fosa axilar moderadamente larga en CRINAHP-1841, presente en los elementos hyoplastrales de Trachemys (Ernst 1990: Fig. 4.4), mientras que la forma y tamaño de CRINAHP-1842 permiten apreciar que se trataba de una tortuga de tamaño mediano, con la característica forma oval y ligeramente arqueada del caparazón de Trachemys (Seidel \& Smith 1986; Ernst \& Barbour 1989).

En cuanto al registro fósil en México, recientemente fueron hallados fragmentos de caparazón de Trachemys para el Pleistoceno de Los Tanques, Zacatecas (Guzmán \& Polaco 2000); Atoyac, Veracruz (Peña-Serrano \& Miranda-Flores 2006) y La Simpatía, Chiapas (Montellano et al. 2008; Luna-Espinosa \& Carbot-Chanona 2009). Otros reportes mexicanos de emydidos fósiles relacionados con Trachemys, son Pseudemys del Mioceno en Potrero Zietla, Hidalgo (Castillo-Cerón et al. 1996; Castillo-Cerón 2000), del Plioceno en Chapala, Jalisco (Smith 1980 cit. por BarriosRivera 1985) y del Pleistoceno en Rancho la Brisca, Sonora (Van Devender et al. 1985); mientras que Chrysemys se ha encontrado en las localidades pleistocénicas Grutas de Coconá en Teapa, Tabasco (Polaco-Ramos 1981) y El Golfo en Sonora (Lindsay 1984). Sin embargo, en una revisión reciente Reynoso (2006) señala que los fósiles reportados como Pseudemys y Chrysemys, en realidad son Trachemys, lo que apoya la relación de los fósiles de Nealtican con dicho género. Cabe señalar que el registro de Trachemys en Nealtican, representa el primer registro fósil de este género para el estado de Puebla (Apéndice 1).

Consideraciones biogeografícas. El área de distribución actual de Trachemys en México se limita a estados del norte y a regiones costeras del Pacífico y del Golfo de México (Ernst 1990; Flores-Villela 1993; Seidel 2002). Sin embargo, pese a ser limitados los registros fósiles de este género, indican que durante finales del Terciario y Cuaternario, Trachemys tuvo una amplia distribución en México (Fig. 4). Por 


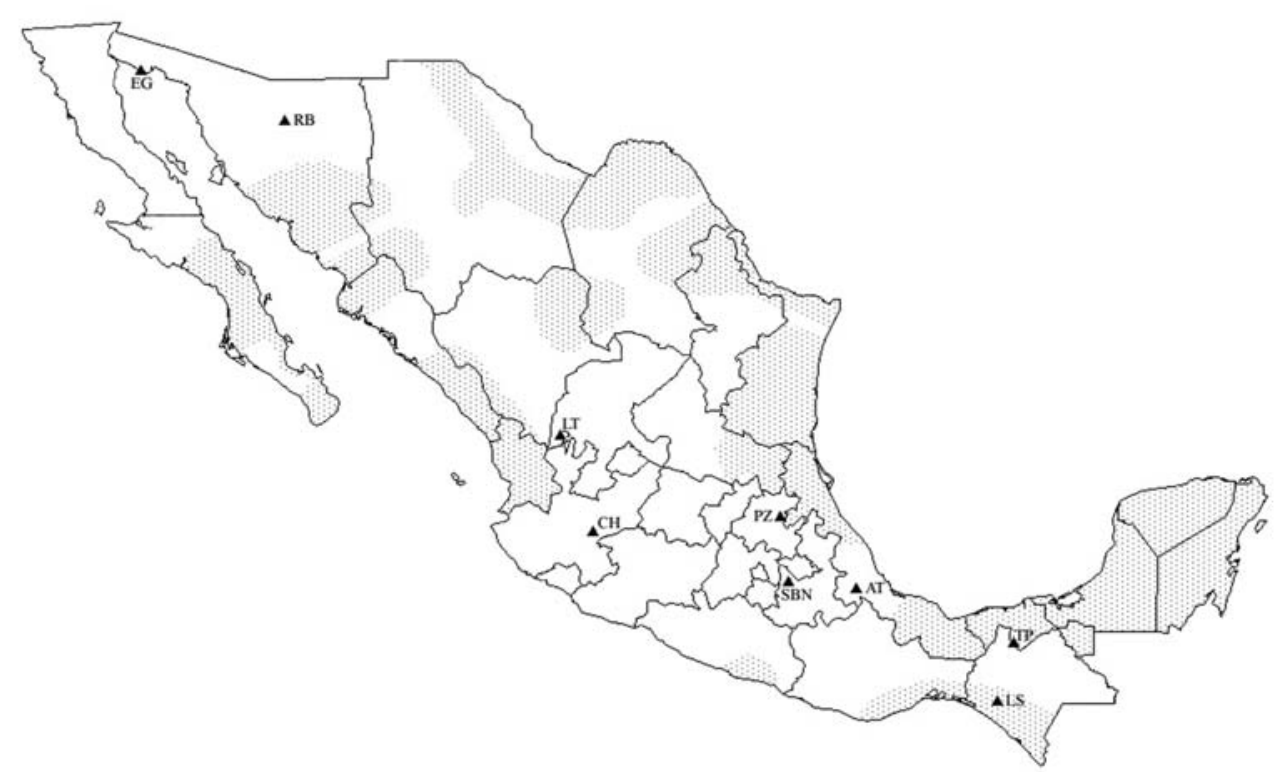

Figura 4. Distribución actual y localidades fósiles de Trachemys en México. Área sombreada = Distribución actual de Trachemys según Seidel (2002). Triángulos = Localidades fósiles para Trachemys. AT = Atoyac; $\mathrm{CH}=$ Chapala $; \mathrm{EG}=\mathrm{El}$ Golfo; $\mathrm{LS}=$ La Simpatía; $\mathrm{LT}=$ Los Tanques; $\mathrm{PZ}=$ Potrero Zietla; $\mathrm{RB}=$ Rancho la Brisca; $\mathrm{SBN}=$ San Buenaventura Nealtican; $\mathrm{TP}=$ Teapa.

ejemplo, los registros en Atoyac, La Simpatía, Potrero Zietla, San Buenaventura Nealtican y Teapa, apuntan que la distribución de Trachemys a finales del Terciario y durante el Cuaternario debió ser continua desde el centro hasta el sur de México, y que posteriormente durante el Holoceno se fragmentó hasta llegar a su forma actual. Por su parte, las localidades de Chapala, El Golfo, Los Tanques y Rancho la Brisca, muestran que durante el Pleistoceno este taxón debió estar distribuido a lo largo de la Sierra Madre Occidental (Fig. 4). Los emydidos (e. g. Trachemys), generalmente están asociados a cuerpos de agua, lo que sugiere que las condiciones ambientales en lugares como Nealtican, El Golfo y Rancho la Brisca fueron muy diferentes de las actuales durante la prehistoria.

AGRADECIMIENTOS. Agradezco al Centro Regional INAH-Puebla, por todas las facilidades otorgadas, en especial a su Director el Antropólogo Víctor Hugo Valencia Valera y al Coordinador de la Sección de Antropología Física el Dr. Zaid Lagunas Rodríguez por su apoyo al hacer disponible el material fósil para su estudio. Al Dr. Víctor Hugo Reynoso Rosales (Instituto de Biología, UNAM), por sus comentarios sobre la identificación taxonómica de los ejemplares. Al Dr. Luis Espinosa Arrubarrena y a la Dra. Ana Luisa Carreño (Instituto de Geología, UNAM), por su amabilidad al facilitarme material 
bibliográfico. Al Dr. Gustavo Aguirre León (Instituto de Ecología A. C.), por sus valiosos comentarios, sugerencias y revisiones del manuscrito. A los revisores anónimos que ayudaron a mejorar el manuscrito.

\section{LITERATURA CITADA}

Arroyo-Cabrales, J., O. J. Polaco \& E. Johnson. 2002. La mastofauna del cuaternario tardío en México. Pp. 103-123. In: M. Montellano-Ballesteros y J. Arroyo-Cabrales (eds). Avances en los estudios paleomastozoológicos. Instituto Nacional de Antropología e Historia, México D. F.

Barrios-Rivera, H. 1985. Estudio analítico del registro paleovertebradológico de México. Tesis de Licenciatura, Universidad Nacional Autónoma de México, México.

Castillo-Cerón, J. M. 2000. Fossil vertebrates from the Miocene of Hidalgo, México. Journal of Vertebrate Paleontology 20: 34A.

Castillo-Cerón, J. M., M. A. Cabral-Perdomo \& O. Carranza-Castañeda. 1996. Vertebrados Fósiles del Estado de Hidalgo. Universidad Autónoma del Estado de Hidalgo, Publicaciones Especiales.

Cruz-Muñoz, V. 2001. Catálogo de vertebrados fósiles del Centro Regional INAH de Puebla. Tesis de Licenciatura, Benemérita Universidad Autónoma de Puebla, México.

Cruz-Muñoz, V., H. E. Castro-Azuara, M. F. Rojas-Cortés \& O. J. Robles-Romero. 1998. Catálogo Paleofaunístico del Centro Regional INAH de Puebla. Pp. C05. En: Memorias del IV Congreso Nacional de Mastozoología. Universidad Veracruzana, Instituto de Ecología A. C. y Sociedad Mexicana de Mastozoología A. C.

Erffa, A. \& W. Hilger. 1977. Geologische karte des gebietes von Puebla-Tlaxcala. In W. Laurer (ed). Geologie des hochbeckens von Puebla-Tlaxcala und seiner umgebung. Franz Steiner Verlage GMBH-Wiesbaden.

Erffa, A., W. Hilger, K. Knoblich \& R. Weyl. 1976. Geología de la Cuenca alta de Puebla-Tlaxcala y sus contornos. Comunicaciones Proyecto Puebla-Tlaxcala 13: 99-117.

Ernst, C. H. 1990. Systematics, taxonomy, variation, and geographic distribution of the slider turtle. Pp. 57-67. In: J. W. Gibbons (ed.). Life History and Ecology of the Slider Turtle. Smithsonian Institution Press, Washington, D C.

Ernst, C. H. \& R. W. Barbour. 1989. Turtles of the World. Smithsonian Institution Press, U.S.A.

Flannery, K. V. 1967. Vertebrate fauna and hunting patterns. Pp. 132-177. In D. S. Byers (ed.). The prehistory of the Tehuacan Valley. University of Texas Press, Austin.

Flores-Villela, O. 1993. Herpetofauna mexicana: Lista anotada de las especies de anfibios y reptiles de México, cambios taxonómicos recientes, y nuevas especies. Carnegie Museum of Natural History Special Publication 17, Pittsburgh.

Guenther, E.W. 1968. Untersuchungen zur jungeiszeitlichen und nacheiszeitlichen geologischen und palaontologisc Geschichte. Pp. 32-37. In Tichy, F. (ed). Das Mexiko projekt der Deutschen Forschungsgemeinschaft-Berichte uber begonnene und geplante arbeiten. Franz Steiner Verlage GMBH-Wiesbaden.

Guenther, E. W. \& H. Bunde. 1973. Investigaciones geológicas y paleontológicas en México durante los años de 1965 a 1969. Comunicaciones Proyecto Puebla-Tlaxcala 7:19-20.

Guillette, D., I. Ferrusquía-Villafranca, E. Jiménez-Hidalgo, O. Gutiérrez-Limón \& G. F. Gunnel. 2000. Preliminary report on a new Pleistocene Faunal site in southern Puebla, México. Journal of Vertebrate Paleontology 20: 44A.

Guzmán, A. F. \& O. J. Polaco. 2000. Vertebrados pleistocénicos de los Tanques, Zacatecas. Pp. 100101. En: Memorias del VII Congreso Nacional de Paleontología. Facultad de Ciencias de la Tierra, U.A.N.L. y Sociedad Mexicana de Paleontología. 
Hay, O. P. 1908. The Fossil turtles of North America. Carnegie Institution of Washington, U.S.A.

Joyce, W. G., J. F. Parham \& J. C. Gauthier. 2004. Developing a protocol for the conversion of rankbased taxon names to phylogenetically defined clade names, as exemplified by turtles. Journal of Paleontology 78: 989-1013.

Lindsay, E. H. 1984. Late Cenozoic mammals from northwestern Mexico. Journal of Vertebrate Paleontology 4: 208-215.

Luna-Espinosa, J. R. \& G. Carbot-Chanona. 2009. Primeros registros de tortugas fósiles para el Pleistoceno tardío de Chiapas, México. Pp. 110. En: Memorias del XI Congreso Nacional de Paleontología. Centro de Geociencias U.N.A.M. y Sociedad Mexicana de Paleontología.

Macías, J. L. 2005. Geología e historia eruptiva de algunos de los grandes volcanes de México. Boletín de la Sociedad Geológica Mexicana 57: 379-424.

Montellano, M., G. Carbot-Chanona, G. Domínguez-Vázquez \& G. Islebe. 2008. Late Pleistocene vertebrate fauna from the Depresión Central of Chiapas, Mexico. Journal of Vertebrate Paleonto$\operatorname{logy} 28: 117 \mathrm{~A}$.

Peña-Serrano, J. \& F. Miranda-Flores. 2006. Hallazgo de un perezoso gigante y fauna asociada del Pleistoceno Tardío en el Municipio de Atoyac, Veracruz, México. Pp. 64. En: Memorias del X Congreso Nacional de Paleontología. Instituto de Geología, U.N.A.M. y Sociedad Mexicana de Paleontología.

Plunket, P. \& G. Uruñuela. 1999. Preclassic household patterns preserverd under volcanic ash at Tetimpa, Puebla, Mexico. Latin America Antiquity 9: 287-309.

Polaco-Ramos, O. J. 1981. Restos fósiles de Glossotherium y Eremotherium (Edentata) en México. Pp. 819-831. En: Anais II Congresso Latino-Americano de Paleontología. Porto Alegre, Brasil.

Reynoso, V. H. 2006. Research on fossil amphibians and reptiles in Mexico, from 1869 to early 2004 (including marine forms but excluding pterosaurs, dinosaurs, and obviosly, birds). Pp. 209-231. In: F. J. Vega, T. G. Nyborg, M. C. Perrilliat, M. Montellano-Ballesteros, S. R. S. Cevallos-Ferriz and S. A. Quiroz-Barroso (eds). Studies on Mexican Paleontology. Springer, The Netherlands.

Seele, E. 1973. Restos de milpas y poblaciones prehispánicas cerca de San Buenaventura Nealtican, Puebla. Comunicaciones Proyecto Puebla-Tlaxcala 7: 77-86.

Siebe, C., J. L. Macias, M. Abrams, S. Rodríguez, R. Castro \& H. Delgado. 1995. Quaternary explosive volcanism and pyroclastic deposits in east central Mexico: implications for future hazards. Guidebook of geological excursions: in conjunction with the Annual Meeting of the Geological Society of America, New Orleans, Louisiana.

Seidel, M. E. 2002. Taxonomic Observations on Extant Species and Subspecies of Slider Turtles, Genus Trachemys. Journal of Herpetology 36: 285-292.

Seidel, M. E. \& H. M. Smith. 1986. Chrysemys, Pseudemys, Trachemys (Testudines: Emydidae): did Agassiz have it right?. Herpetologica 42(2): 242-248.

Tovar-Liceaga, R. E. \& M. Montellano-Ballesteros. 2004. Microfauna pleistocénica de Santa Cruz Nuevo, Puebla. P. 61. En: Memorias del IX Congreso Nacional de Paleontología. Instituto de Historia Natural de Chiapas y Sociedad Mexicana de Paleontología.

Tovar-Liceaga, R. E. \& M. Montellano-Ballesteros. 2005. Pleistocene fauna from Santa Cruz Nuevo, Puebla, central México. Journal of Vertebrate Paleontology 25: 123A.

Tovar, R. E., M. Montellano-Ballesteros \& E. Corona-M. 2007. Fauna Pleistocénica de Santa Cruz Nuevo, Puebla. Pp. 393-397. In: E. Diaz-Martinez e I. Rabano (eds). 4th European Meeting on the Paleontology and Stratigraphy of Latin America. Instituto Geológico y Minero de España, Madrid.

Van Devender, T. R., A. Rea \& M. L. Smith. 1985. The Sangamon interglacial vertebrate fauna from Rancho la Brisca, Sonora, México. Transactions of the San Diego Society of Natural History 21:23-55. 
Herrera Flores: Tortugas fósiles en Nealtican, Puebla

Apéndice 1. Localidades con herpetofauna de edad pleistocénica en el estado de Puebla.

A. Cañón Tecorral en el Valle de Tehuacán (Flannery 1967):

Reptilia

Testudines

Cryptodira

Testudinidae

Gopherus berlandieri

B. Puente Atepetzingo en Valsequillo (Guenther 1968):

Reptilia

Testudines

Cryptodira

Testudinidae

Testudo sp.

C. Santa Cruz Nuevo, Municipio de Totoltepec de Guerrero (Guillette et al. 2000; Tovar-Liceaga \& Montellano-Ballesteros 2004, 2005; Tovar et al. 2007):

Amphibia

Caudata

Ambystomatidae

Ambystoma sp.

Plethodontidae gen. et sp. indet.

Anura

Bufonidae

Bufo occidentalis

Bufo cf. valliceps

Bufo sp.

Ranidae

Rana sp.

Scaphiopodidae gen. et sp. indet.
Reptilia

Testudines

Cryptodira

Testudinidae

Gopherus cf. berlandieri

Kinosternidae

Kinosternon sp.

Bataguridae

Rhinoclemmys sp.

Squamata

Lacertilia

Phrynosomatidae

Sceloporus cf. grammicus

Sceloporus $\mathrm{cf}$. horridus

Scincidae gen. et sp. indet.

Serpentes

Colubridae

Conopsis sp.

Lampropeltis sp.

Leptodeira sp.

Senticolis sp.

Thamnophis sp.

Trimorphodon sp.

Viperidae

Crotalus sp.. 